OPEN ACCESS

Edited by:

Thomas Rawson,

University of Oxford, United Kingdom

Reviewed by:

Vahé Nafilyan,

Office for National Statistics,

United Kingdom

Lihua Zhu,

Wuhan University, China

*Correspondence:

Daniel Kondziella

daniel.kondziella@regionh.dk

Michael E. Benros

michael.eriksen.benros@regionh.dk

tThese authors have contributed equally to this work and share first authorship

*These authors have contributed equally to this work and share senior authorship

Specialty section:

This article was submitted to Infectious Diseases - Surveillance,

Prevention and Treatment, a section of the journal

Frontiers in Medicine

Received: 24 August 2020 Accepted: 04 November 2020

Published: 30 November 2020

Citation:

Nersesjan V, Amiri M, Christensen HK, Benros ME and Kondziella D (2020)

Thirty-Day Mortality and Morbidity in COVID-19 Positive vs. COVID-19

Negative Individuals and vs. Individuals Tested for Influenza A/B: A

Population-Based Study.

Front. Med. 7:598272.

doi: 10.3389/fmed.2020.598272

\section{Thirty-Day Mortality and Morbidity in COVID-19 Positive vs. COVID-19 Negative Individuals and vs. Individuals Tested for Influenza A/B: A Population-Based Study}

\author{
Vardan Nersesjan ${ }^{1 \dagger}$, Moshgan Amiri ${ }^{1 \dagger}$, Hanne K. Christensen ${ }^{2,3}$, Michael E. Benros ${ }^{4,5 *}$ and \\ Daniel Kondziella ${ }^{1,3 * \neq}$ \\ ${ }^{1}$ Department of Neurology, Rigshospitalet, Copenhagen University Hospital, Copenhagen, Denmark, ${ }^{2}$ Department of \\ Neurology, Bispebjerg Hospital, Copenhagen University Hospital, Copenhagen, Denmark, ${ }^{3}$ Department of Clinical Medicine, \\ Faculty of Health and Medical Sciences, University of Copenhagen, Copenhagen, Denmark, ${ }^{4}$ Copenhagen Research Centre \\ for Mental Health - CORE, Mental Health Centre Copenhagen, Copenhagen University Hospital, Hellerup, Denmark, \\ ${ }^{5}$ Department of Immunology and Microbiology, Faculty of Health and Medical Sciences, University of Copenhagen, \\ Copenhagen, Denmark
}

Background: As of October 2020, COVID-19 has caused 1,000,000 deaths worldwide. However, large-scale studies of COVID-19 mortality and new-onset comorbidity compared to individuals tested negative for COVID-19 and individuals tested for influenza A/B are lacking. We investigated COVID-19 30-day mortality and new-onset comorbidity compared to individuals with negative COVID-19 test results and individuals tested for influenza $A / B$.

Methods and findings: This population-based cohort study utilized electronic health records covering roughly half $(n=2,647,229)$ of Denmark's population, with nationwide linkage of microbiology test results and death records. All individuals $\geq 18$ years tested for COVID-19 and individuals tested for influenza A/B were followed from 11/2017 to $06 / 2020$. Main outcome was 30-day mortality after a test for either COVID-19 or influenza. Secondary outcomes were major comorbidity diagnoses 30-days after the test for either COVID-19 or influenza A/B. In total, 224,639 individuals were tested for COVID-19. To enhance comparability, we stratified the population for in- and outpatient status at the time of testing. Among inpatients positive for COVID-19, 356 of 1,657 (21\%) died within 30 days, which was a 3.0 to 3.1-fold increased 30-day mortality rate, when compared to influenza and COVID-19-negative inpatients (all $p<0.001$ ). For outpatients, 128 of 6,263 (2\%) COVID-19-positive patients died within 30 days, which was a 5.5 to 6.9-fold increased mortality rate compared to individuals tested negative for COVID-19 or individuals tested positive or negative for influenza, respectively (all $p<0.001$ ). Compared to hospitalized patients with influenza $A / B$, new-onset ischemic stroke, diabetes and nephropathy occurred more frequently in inpatients with COVID-19 (all $p<0.05$ ).

Conclusions: In this population-based study comparing COVID-19 positive with COVID-19 negative individuals and individuals tested for influenza, COVID-19 was 
associated with increased rates of major systemic and vascular comorbidity and substantially higher mortality. Results should be interpreted with caution because of differences in test strategies for COVID-19 and influenza, use of aggregated data, the limited 30-day follow-up and the possibility for changing mortality rates as the pandemic unfolds. However, the true COVID-19 mortality may even be higher than the stated 3.0 to 5.5-fold increase, owing to more extensive testing for COVID-19.

Keywords: COVID-19, SARS-CoV-2, coronavirus, ischemic heart disease, morbidity, mortality, neurology, psychiatry

\section{INTRODUCTION}

COVID-19 has led to a worldwide healthcare crisis with $>30,000,000$ confirmed infected people, resulting in $1,000,000$ deaths as of October $2020(1,2)$. Governmental initiatives including lockdowns and social distancing are aiming to restrict the spread of the virus. Yet, critical voices (3) have argued the socioeconomic consequences may be unjustified given that little is known about how the pandemic compares with annual influenza epidemics in terms of mortality and morbidity. According to the WHO seasonal influenza $\mathrm{A} / \mathrm{B}$ may result in 290,000-650,000 deaths worldwide annually $(4,5)$. Substantially higher mortality rates for COVID-19 will result in even more adverse impact on global health without strict preventive measures. However, large-scale studies including follow-up of individuals tested for COVID-19 and influenza A/B from the same cohort are lacking.

Of further concern, COVID-19 might not only be a respiratory disease but a multi-organ disorder because of the wide expression of the angiotensin-converting enzyme-2 receptor to which SARS-Cov-2 binds (6), leading among others to thromboembolic complications (7), severe inflammatory responses (8), and possibly diabetes (9). Neurological and psychiatric complications will likely constitute a major health burden as well $(10,11)$. But how COVID-19 morbidity compares to similarly severe influenza morbidity is equally poorly understood.

Here, for the first time, we utilized population-based electronic health records (EHR) from Denmark linked with nationwide databases on test results for infections and death records, to investigate mortality in people with COVID-19 compared to people with influenza and to people tested negative for COVID-19. For secondary outcomes we estimated COVID19-associated new-onset comorbidity, including cardiovascular, neurological and psychiatric events, compared to influenza and individuals tested COVID-19-negative. Analyses were stratified according to age, sex and in- and outpatient status. We hypothesized that COVID-19 would be associated with higher mortality and increased rates of novel comorbidities compared to influenza $\mathrm{A} / \mathrm{B}$.

\section{MATERIALS AND METHODS}

This retrospective Danish study was based on EHR covering two well-defined administrative regions: Capital Region (i.e., Greater
Copenhagen and Bornholm) and Region Zealand, comprising roughly $50 \%$ of the Danish population. Denmark has an almost exclusively public health care sector based on catchment areas.

\section{Registers and Study Population}

The EHR system of the Capital and Zealand Regions, which is called EPIC (version 2019, Verona, Wisconsin, USA), consists of data from all hospital contacts in these regions. From implementation in 2016 to June 30, 2020, 2,647,229 individuals were registered. Diagnoses are defined according to ICD-10 (12). Registration of death in the EHR is synchronized with the Danish national population registry, updated every $24 \mathrm{~h}$. Accuracy of test results for influenza and SARS-CoV-2 virus is ensured by synchronization of EPIC with the nationwide Danish Microbiology Database (13). All individuals $\geq 18$ years tested for COVID-19 between March 1-June 1, 2020, and all individuals tested for influenza $\mathrm{A} / \mathrm{B}$ between November 1, 2017-June 1, 2020, were followed for mortality and newonset comorbidities 30-days after the test until June 30, 2020. Included individuals in this study were hospitalized patients who were tested for COVID-19 or influenza during admission (from now on referred to as inpatients); and non-hospitalized patients screened during ambulatory visit, as well as healthy individuals screened in hospital-based testing facilities created for the purpose of screening the general population (from now on referred to as outpatients).

\section{Assessment of COVID-19 and Influenza Test Results COVID-19}

All individuals tested for COVID-19 during March 1-June 1, 2020 with laboratory tests CORONAVIRUS 2019-NCOV and/or CORONAVIRUS SARS-COV-2 RNA via nasal, pharyngeal and/or tracheal samples with reverse-transcriptase-polymerasechain reaction (RT-PCR) assays were included. These specific tests cover all performed COVID-19 tests in the catchment areas and are available from the Danish Microbiology Database (13).

\section{Influenza A/B}

We included all individuals tested for influenza $\mathrm{A} / \mathrm{B}$ during November 1, 2017 to March 1, 2020, using 9 different RTPCR laboratory tests (Supplementary Table 1), covering all available influenza tests based on nasal, pharyngeal and/or tracheal samples. 


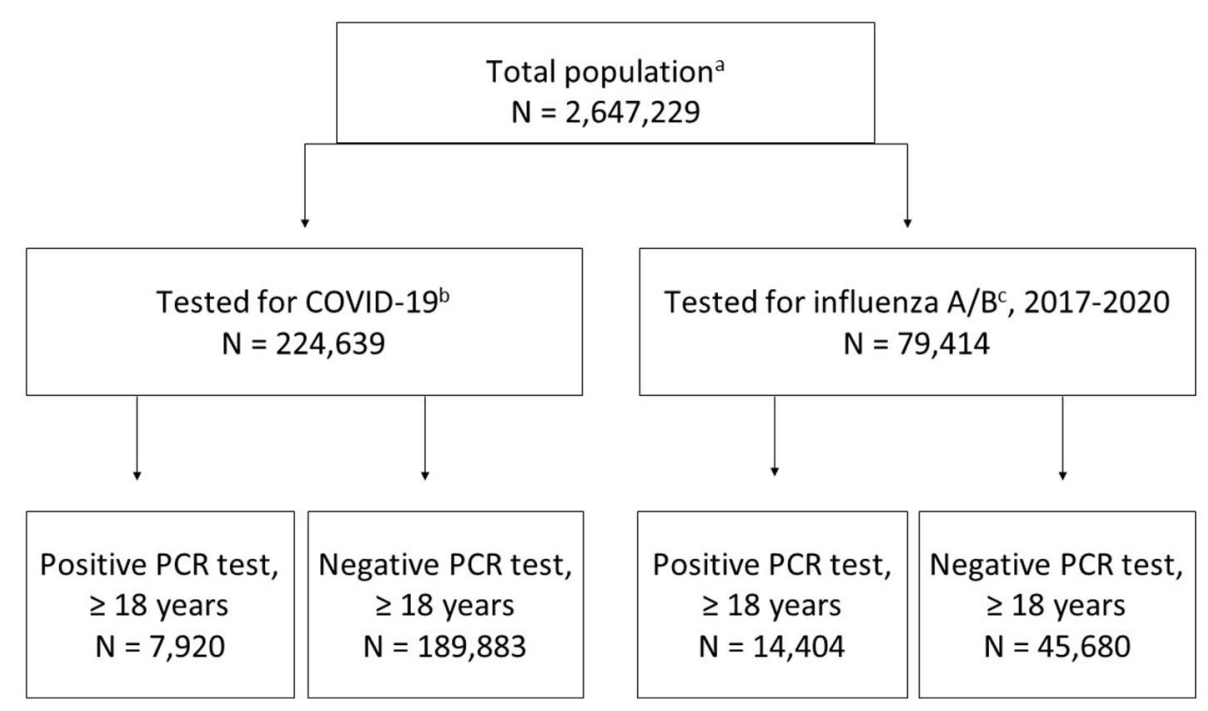

FIGURE 1 | Flow chart of study populations. (a) total population in EHR registered per June 30, 2020; (b) tested between March 1-June 1, 2020; (c) tested between November 1, 2017-March 1, 2020.

\section{Outcome Measures}

\section{Primary Outcome Measures}

Thirty-day mortality among the group of inidividuals tested positive for COVID-19, compared to 30-day mortality of the group of individuals with COVID-19-negative tests. Additional comparisons were made to the group of individuals tested influenza-positive or influenza-negative.

\section{Secondary Outcome Measures}

New-onset (i.e., 30 days after COVID-19 or influenza test) comorbidity diagnoses, including neurological, psychiatric and cardiovascular disease, pulmonary embolism, venous thrombosis, renal failure, diabetes and rheumatoid arthritis, in all populations. ICD-10 codes are listed in Supplementary Table 1.

\section{Data Collection, Statistical Analysis, and Ethics}

Anonymized retrospective aggregate-level data on sex, age, prior comorbidities and population mortality 30 days after test results were extracted for individuals $\geq 18$ years for each groups, using the EPIC Slicer-Dicer function. For search strategies see Supplementary Table 1 . As individuals could be tested multiple times, individuals were only included in the COVID-19-negative, respectively, influenza-negative populations, when all their tests had been negative. Individuals tested for influenza during March 1-June 1, 2020 (i.e., FLU-19) were included for sensitivity analysis (see below). To avoid overlap, we removed COVID-19-positive individuals from the FLU-19 group.

Main analysis was the relative risk (RR) of mortality rates 30 days after a test, in the overall populations and stratified according to in- and outpatient status, sex, and age. Secondary analysis was $\mathrm{RR}$ of cumulative 30 days post-test incidence of new-onset comorbidities, after exclusion of individuals who already had the investigated comorbidity before the test.
We compared COVID-19 positive with COVID-19-negative and influenza-positive individuals. To validate mortality data, absolute mortality rates extracted from electronic health records (EPIC) were compared with official Danish statistics numbers (Supplementary Table 2). Sensitivity analysis was conducted by comparing individuals $\geq 18$ years with a positive or negative influenza test from the same time period as the COVID-19 population, i.e., March 1-June 1, 2020 (FLU-19), in order to investigate the possible influence of the COVID-19 pandemic, including lockdown and social distancing measures, on mortality rates in individuals tested for influenza. Chi-squared statistics were used to calculate odds ratio (OR), RR and 95\% confidence intervals (CI) using SPSS (version 25; IBM, Armonk, NY, USA). Two-sided $p \leq 0.05$ was considered significant.

The Ethics Committee of the Capital Region of Denmark waives approval for register-based studies on aggregated anonymized data (Section 14.2 of the Committee Act. 2; http:// www.nvk.dk/english). Use of anonymized aggregate-level data was approved by the Danish Data Protection Agency. Results from $\leq 5$ patients were displayed as " $\leq 5$ " to ensure data privacy.

\section{RESULTS}

A total of 224,639 individuals of any age were tested for SARSCoV-2 between March 1-June 1, 2020; positive results were found in 7,920 individuals $\geq 18$ years (i.e., our case population). A negative COVID-19 test occurred in 189,883 individuals $\geq 18$ years. Between November 1, 2017-March 1, 2020, we identified 79,414 individuals, who were tested for influenza A/B. Positive results were found in 14,404 individuals aged $\geq 18$ years. Negative influenza A/B tests were identified in 45,680 individuals $\geq 18$ years (Figure 1). Demographics are displayed in Table 1 and Supplementary Tables 3-5. The proportion of inpatients at the time of COVID-19 or influenza tests was lower 
TABLE 1 | Demographics and prior comorbidities among individuals tested for COVID-19 or influenza as in- or outpatient.

\begin{tabular}{|c|c|c|c|c|c|c|}
\hline & \multicolumn{3}{|c|}{ Inpatients } & \multicolumn{3}{|c|}{ Outpatients } \\
\hline & $\begin{array}{l}\text { CovID-19 positive } \\
\qquad(N=1,657)\end{array}$ & $\begin{array}{l}\text { COVID-negative } \\
\qquad(N=31,483)\end{array}$ & $\begin{array}{l}\text { Influenza-positive } \\
(N=7,200)\end{array}$ & $\begin{array}{l}\text { CovID-19-positive } \\
\quad(N=6,263)\end{array}$ & $\begin{array}{l}\text { COVID-negative } \\
(N=158,400)\end{array}$ & $\begin{array}{l}\text { Influenza-positive } \\
\qquad(N=7,204)\end{array}$ \\
\hline \multicolumn{7}{|l|}{ Age - years, no. (\%) } \\
\hline Mean, years & 65 & 60 & 66 & 47 & 48 & 49 \\
\hline $18-39$ & $182(11.0)$ & $7,456(23.7)^{\ddagger}$ & $931(12.9)$ & 2,310 (36.9) & $54,659(34.5)^{\ddagger}$ & $2,390(33.2)^{\ddagger}$ \\
\hline $40-59$ & $427(25.8)$ & $6,790(21.6)^{\ddagger}$ & $1,413(19.6)^{\ddagger}$ & $2,509(40.1)$ & $62,701(39.6)$ & $2,637(36.6)^{\ddagger}$ \\
\hline $60-80$ & $636(38.4)$ & $11,261(35.8)^{\ddagger}$ & $2,876(39.9)$ & $1,043(16.7)$ & $33,166(20.9)^{\ddagger}$ & $1,76224.4)^{\ddagger}$ \\
\hline$>80$ & $412(24.9)$ & $5,976(19.0)^{\ddagger}$ & $1,980(27.5)$ & $401(6.4)$ & $7,874(5.0)^{\ddagger}$ & $415(5.8)$ \\
\hline \multicolumn{7}{|l|}{ Sex (\%) } \\
\hline Women & $737(44.5)$ & $17,099(54.3)^{\ddagger}$ & $3,844(53.4)^{\ddagger}$ & 3,931 (62.8) & $99,364(62.7)$ & $4,272(59.3)^{\ddagger}$ \\
\hline \multicolumn{7}{|c|}{ Prior medical diagnoses - no. (\%) } \\
\hline Neurological, any & $275(16.6)$ & 4,998 (15.9) & $1,025(14.2)$ & $505(8.1)$ & $14,074(8.9)^{\ddagger}$ & $474(6.6)^{\ddagger}$ \\
\hline Cerebrovascular, any & $141(8.5)$ & $2,819(9.0)$ & $485(6.7)$ & $145(2.3)$ & 3,698 (2.3) & $125(1.7)^{\ddagger}$ \\
\hline Ischemic stroke incl. TIA & $78(4.7)$ & $1,739(5.5)$ & $165(2.3)^{\ddagger}$ & $85(1.4)$ & $2,302(1.5)$ & $130(1.8)^{\ddagger}$ \\
\hline Psychiatric, any & 202 (12.2) & $6,581(20.9)^{\ddagger}$ & $821(11.4)$ & $383(6.1)$ & $12,264(7.7)^{\ddagger}$ & $305(4.2)^{\ddagger}$ \\
\hline Ischemic heart disease & $147(8.9)$ & $2,631(8.4)$ & $547(7.6)$ & $90(1.4)$ & $3,719(2.3)^{\ddagger}$ & $155(2.2)^{\ddagger}$ \\
\hline Heart failure & $100(6.0)$ & $1,861(5.9)$ & $422(5.9)$ & $45(0.7)$ & $1,581(1.0)^{\ddagger}$ & $68(0.9)$ \\
\hline Diabetes & $199(12.0)$ & $3,291(10.5)^{\ddagger}$ & $827(11.5)$ & 209 (3.3) & $4,903(3.1)$ & 255 (3.5) \\
\hline Chronic lower respiratory disease & $182(11.9)$ & $3,943(12.5)$ & $1,362(18.9)^{\ddagger}$ & $223(3.6)$ & $7,934(5.0)^{\ddagger}$ & $366(5.1)^{\ddagger}$ \\
\hline Obesity & $46(2.8)$ & $1,296(4.1)^{\ddagger}$ & $167(2.3)$ & $132(2.1)$ & $3,929(2.5)$ & $141(2.1)$ \\
\hline
\end{tabular}

COVID-19, positive COVID-19 PCR test between March 1-June 1, 2020; influenza, positive influenza A/B PCR test between November 1, 2017 to March 1, 2020; COVID-neg, negative COVID-19 PCR test between March 1-June 1, 2020. Each patient was followed for a total of 30 days from positive test until end of follow-up or death. ¥Indicates statistically significant difference $(p<0.05)$ compared to COVID-19 populations. "Established medical diagnoses, registered in the medical files, prior to testing for COVID-19 or influenza. TIA, transitory ischemic attack.

in the COVID-19-positive (20.9\%) and the COVID-19-negative (16.6\%) populations compared to influenza-positive (50\%) and influenza-negative $(57.7 \%)$ populations. We therefore analyzed mortality and comorbidities both in the overall populations and stratified according to in- and outpatient status, sex, and age.

\section{Primary Outcome: Mortality Overall Mortality Rates}

Overall 30-day mortality in COVID-19-positive individuals was 484 of 7,920 (6.1\%), whereas 30-day mortality for COVID-19negative individuals was 2,654 of 189,883 (1.4\%), corresponding to an increased mortality by RR 4.37 ( $95 \% \mathrm{CI}=3.98-4.80)$.

\section{Mortality Rates of Inpatients Tested for COVID-19 and/or Influenza}

Thirty-day mortality for hospitalized COVID-19 patients $\geq 18$ years was 356 of 1,657 (21.5\%), which was higher than in COVID19-negative individuals (30-day mortality 2,185/31,483; 6.9\%; $p$ $<0.001$ ) (Figure 2, Table 2, and Supplementary Tables 6-9). The corresponding numbers for individuals tested positive for influenza were 516/7,200 (7.2\%) and for influenza-negative individuals 2,873/26,366 (11\%). Mortality for COVID-19positive inpatients was increased by RR $3.10(95 \% \mathrm{CI}=2.80$ 3.42) compared to COVID-19-negative patients, and by RR $3.00(95 \% \mathrm{CI}=2.65-3.39)$ and RR $1.97(95 \% \mathrm{CI}=1.79-2.18)$ compared to influenza-positive, respectively, influenza-negative inpatients (all $p<0.001$ ).
When mortality rates were stratified according to age, 30-day mortality rates for hospitalized COVID-19 patients were $16 / 427$ (3.7\%, age 40-59 years), $150 / 636$ (23.6\%, 6080 years) and 190/412 (46\%, >80 years). The corresponding numbers for COVID-19-negative individuals were 158/6,790 (2.3\%), $1,004 / 11,261(8.9 \%)$, and $1,008 / 5,976(16.9 \%)$ and for influenza-positive individuals $26 / 1,413$ (1.8\%), 214/2,876 (7.4\%), and $271 / 1,980$ (13.7\%), respectively. Mortality for COVID-19positive inpatients was significantly increased with age 60-80 years $(\mathrm{RR}=2.65 ; 95 \% \mathrm{CI}=2.27-3.08)$ and $>80$ years $(\mathrm{RR}=3.17$; 95\% CI $=2.62-3.83)$, when compared to COVID-19-negative individuals (RR 2.73; 95\% CI $=2.42-3.08$ ) and influenzapositive individuals ( $\mathrm{RR} 3.37$ (95\% CI $=2.89-3.92)$. When mortality rates were stratified according to sex, 30-day mortality rates for hospitalized COVID-19 patients were 143/738 (19.4\%, female) and 213/919 (23.2\%, male). The corresponding numbers for COVID-19-negative individuals were 1,027/17,134 (6.0\%, female) and 1,158/14,349 (8.1\%, male) and for influenza-positive individuals 240/3,851 (6.2\%, female) and 276/3,349 (8.2\%, male). Mortality for COVID-19-positive inpatients was significantly increased in females and males compared to COVID-19-negative females (RR 3.23; 95\% CI = 2.76-3.79) and males (RR 2.87; 95\% CI $=2.52-3.27$ ) and influenza-positive females (RR 3.11; $95 \% \mathrm{CI}=2.57-3.77)$ and males (RR 2.81; 95\% CI $=2.39$ 3.31). See Table 2 and Supplementary Table 6 for a full outline of inpatient mortality rates stratified according to sex and age groups. 


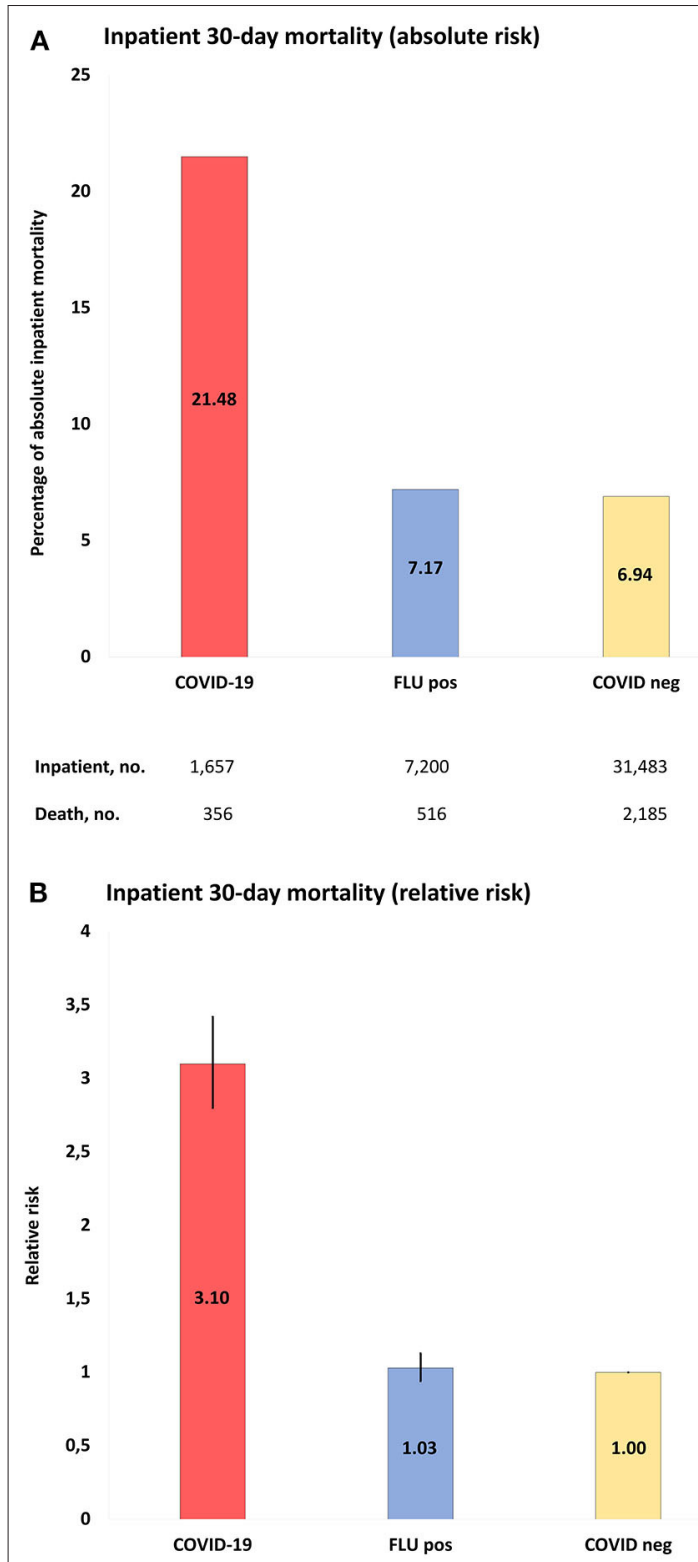

FIGURE 2 | (A), Absolute risk with 95\% Cl of COVID-19 inpatient 30-day mortality, when compared to populations of the study: COVID-19, positive COVID-19 test between March 1-June 1, 2020; FLU pos, positive influenza A/B test between November 1, 2017-March 1, 2020; COVID-neg, negative COVID-19 test between March 1-June 1, 2020. (B), RR with 95\% Cl of inpatient mortality of study populations compared to COVID-19 negative population as reference.

\section{Mortality Rates of Outpatients Tested for COVID-19 and/or Influenza}

Regarding outpatients, positive COVID-19 tests were associated with 128 deaths in 6,263 people ( $2 \% 30$-day mortality) and negative COVID-19 tests with 469 deaths in 158,400 people $(0.3 \%)$, whereas the corresponding numbers for influenzatested people were $27 / 7,204$ (0.4\%; positive test) and 129/19,314 (0.7\%; negative test). Mortality rates for COVID-19-positive outpatients were increased by RR 6.90 (95\% CI = 5.69-8.38) compared to COVID-19-negative outpatients, by RR 5.45 (95\% $\mathrm{CI}=3.61-8.25)$ compared to influenza-positive outpatients, and by RR 3.06 (95\% CI = 2.40-3.90) compared to influenzanegative outpatients. Figure 3 and Supplementary Tables 6-9 show details.

The 30-day mortality rates for outpatients with COVID-19 were $20 / 62,701(0.03 \%), 33 / 1,043(3.2 \%)$, and 92/401 (22.9\%) for age groups $40-59,60-80$, and $>80$ years, respectively. The corresponding numbers for COVID-19 negative individuals were $161 / 33,166(0.5 \%)$ and $288 / 7,874(3.7 \%)$ in the age groups $60-80$ and $>80$ years, and for influenza-positive individuals $\leq 5 / 1,761$ and $20 / 415(4.8 \%)$, respectively. The case numbers were too low in the remaining age groups for statistics. The 30-day mortality rates for outpatients with COVID-19 were 75/3,937 $(1.9 \%)$ and $53 / 2,326(2.3 \%)$ in females and males, respectively. The corresponding numbers for COVID-19 negative individuals were $261 / 99,512(0.3 \%)$ and $208 / 58,888$ (0.4\%) for females and males, respectively, and for influenza-positive individuals $17 / 4,284(0.4 \%)$ and $10 / 2,920(0.3 \%)$, respectively. Outpatient 30-day mortality was significantly increased in COVID-19 males and females compared to COVID-19 negative and Influenza-positive and negative individuals. See Table 2 and Supplementary Table 6 for full details of outpatient mortality rates stratified according to age and sex.

\section{Secondary Outcomes: New-Onset Comorbidities}

Figure 4 and Supplementary Tables 10, 11 display data regarding novel diagnoses after COVID-19 and influenza tests.

\section{New-Onset Comorbidities Among COVID-19-Positive and COVID-19-Negative Individuals}

Pulmonary embolism 30 days after testing was more frequent in COVID-19-positive compared to COVID19-negative individuals [RR 2.47 (95\% CI $=1.60-3.78)$ ], Supplementary Table 10. Diabetes and renal failure were also more frequent in COVID-19-positive compared to negative individuals ( 0.6 vs. $0.2 \%$ and 0.6 vs. $0.1 \%$, respectively; both $p<0.001$ ). Neurological disorders (excluding vascular disorders) and ischemic heart disease were less frequent in COVID-19-positive than in COVID-19-negative people $(0.2$ vs. $0.5 \%$ and 0.1 vs. $0.3 \%$, respectively; both $p<0.05)$. Rates of new-onset cerebrovascular disorders, venous thrombosis and psychiatric disorders were not significantly different between the two populations.

\section{New-Onset Comorbidities in Inpatients Tested Positive for COVID-19 vs. Influenza-Positive Individuals}

Incident ischemic stroke 30 days after a test was more frequent in COVID-19-positive inpatients compared to those with influenza, RR 3.10 (95\% CI = 1.56-6.08), Supplementary Table 11. Newonset diabetes and nephropathy were more frequent in COVID19 positive compared to influenza-positive inpatients (1.9 vs. $1.2 \%$ and 1.8 vs. $0.9 \%$, respectively; both $p<0.05)$. Rates 
TABLE 2 | Relative risk of 30-day mortality after a COVID-19 or influenza test among in- or outpatients.

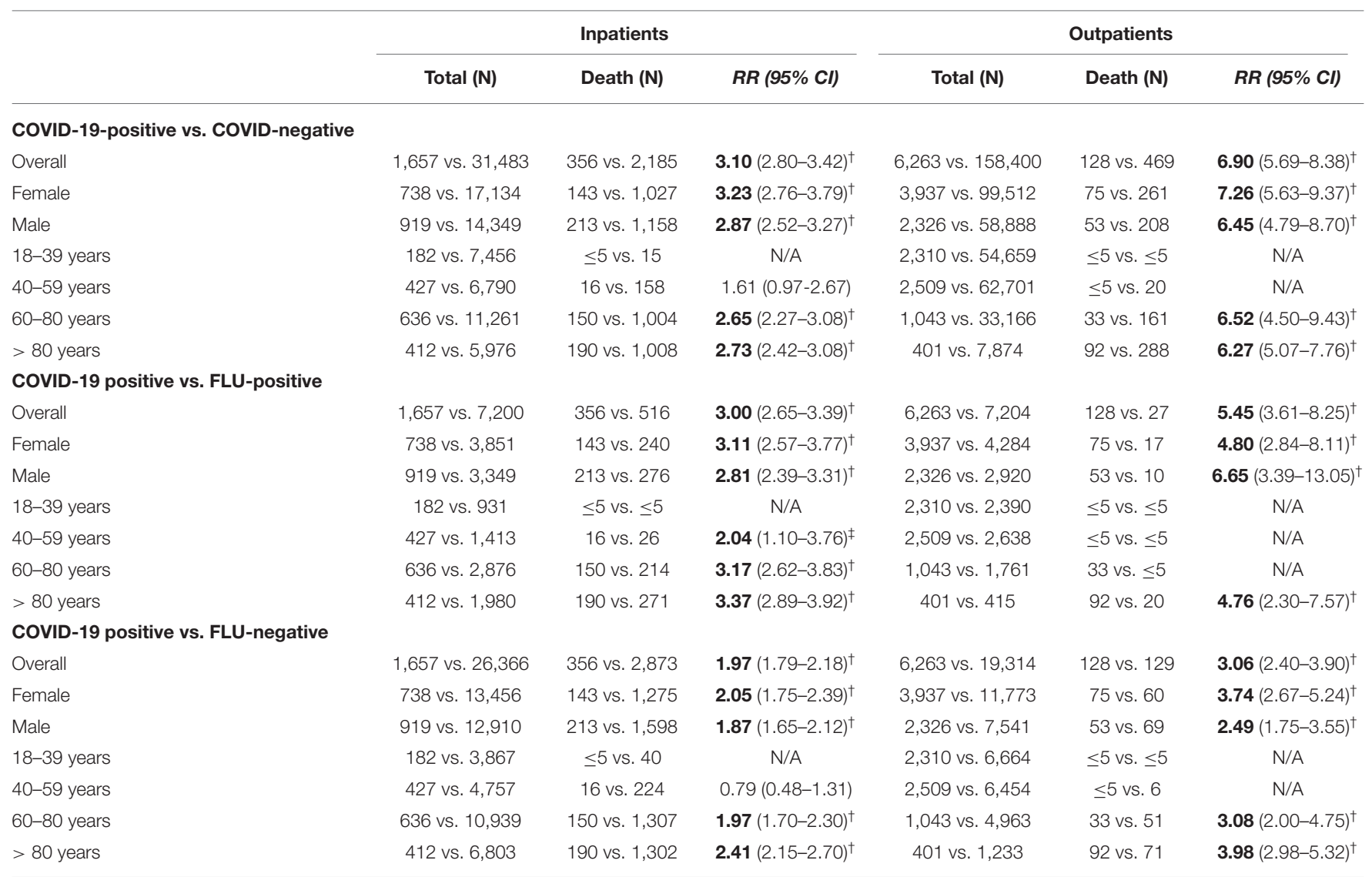

COVID-19, positive COVID-19 test between March 1-June 1, 2020; COVID-neg, negative COVID-19 test between March 1-June 1, 2020; FLU positive, positive influenza A/B test between November 1, 2017-March 1, 2020; FLU negative, negative influenza A/B test between November 1, 2017-March 1, 2020. Each included patient was followed for a total of 30 days from positive test until end of follow-up or death. N/A, not applicable due to low number of patients $(N \leq 5) .{ }^{\dagger} p<0.001 ;{ }^{\ddagger} p<0.05$. The bold values indicate statistically significant values.

of new-onset pulmonary embolism, neurological disorders and psychiatric disorders were not statistically different.

\section{New-Onset Comorbidities in Outpatients Tested Positive for COVID-19 vs. Influenza-Positive Individuals}

Incidence diagnoses 30 days after positive tests in outpatients yielded either too low numbers for meaningful statistics or were not statistically different (Supplementary Table 11).

\section{Sensitivity Analysis}

The COVID-19-positive population was compared to a population of influenza-tested individuals from the same time period, March 1-June 1, 2020, i.e., outside the influenza peak season (FLU-19). In total, 12,502 people were tested for influenza $\mathrm{A} / \mathrm{B}$ (56\% inpatients; 566 positive and 8,318 negative). Inpatient mortality in FLU-19-positive and -negative populations was 26/317 (8.2\%), respectively, 578/5,058 (11.4\%). Inpatient mortality was significantly increased in COVID-19 compared to FLU-19-positive and -negative individuals (RR 2.62 (95\% CI $=1.79-3.83)$, respectively, RR 1.88 (95\% CI = 1.67-2.12); both $p<0.001)$.

\section{DISCUSSION}

To our knowledge, this is the first population-based study comparing mortality rates and new-onset comorbidities of COVID-19 patients with those of COVID-19-negative controls and individuals tested for influenza A/B. 30day mortality was 3.0 to 6.9-fold higher in the group of individuals tested positive for COVID-19 compared to individuals tested COVID-19 negative and when compared with individuals tested for influenza. The largest difference in mortality between COVID-19 and influenza was observed in outpatients. Equally important, new-onset ischemic stroke, renal failure and diabetes occurred at increased rates in COVID-19-positive inpatients compared to influenza patients.

Previous studies have reported widely varying overall COVID19 mortality rates, e.g., $1.4 \%$ among 1,099 cases in Wuhan, China (14), and $7.2 \%$ among 22,512 in Italy (15). In our study, the overall 30-day COVID-19 mortality was $6.1 \%$ and males over 60 years of age were overrepresented, which is well in line with previous data from COVID-19 patients from Denmark (16). Importantly, mortality rates are very different among in- 


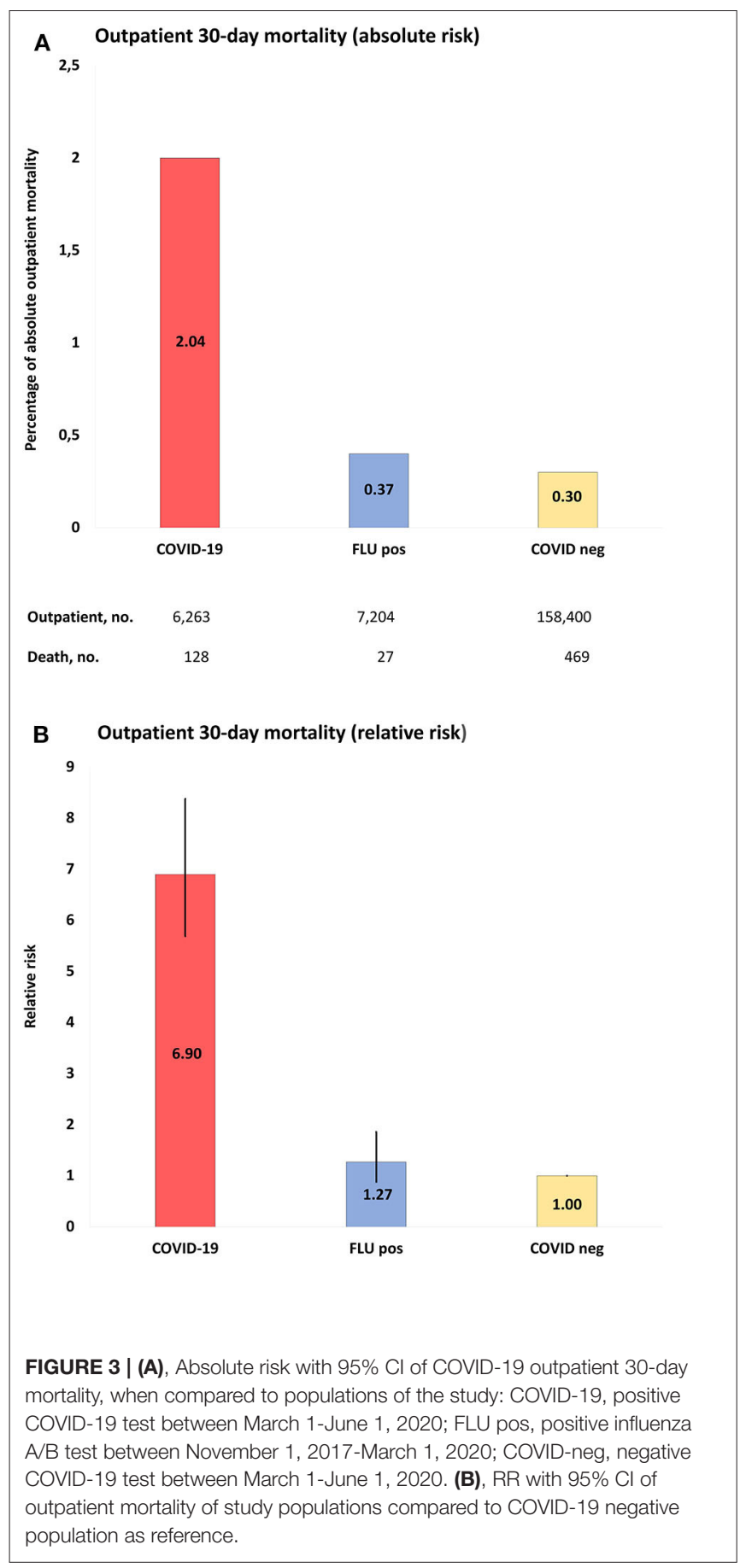

and outpatients. In the COVID-19-positive inpatient population 30 -day mortality was $21 \%$, corresponding well-again with a mortality of $28 \%$ in 191 inpatients reported by Zhou et al. (17), respectively, a median 14-day mortality of $26 \%$ in 140 inpatients from Xie et al. (18). These numbers are much higher than the $2 \%$ mortality in outpatients tested COVID-19 positive (i.e., individuals from the general public not requiring hospitalization) in the present study, indicating that, not surprisingly, inpatients with COVID-19 are doing worse than outpatients.
Compared to individuals tested positive for influenza and individuals tested COVID-19-negative, COVID-19 30-day mortality was increased 3.0 to 3.10 -fold for inpatients and 5.5 to 6.9-fold for outpatients. This is somewhat in contrast with an estimated 20-fold mean increase of COVID-19 mortality compared to influenza, based on indirect estimated numbers from the general public in the US (19). This discrepancy could be explained by the higher proportion of sick individuals in our influenza tested populations, as the influenza testing in Denmark is primarily done on individuals at risk due to chronic conditions. If testing for influenza $\mathrm{A} / \mathrm{B}$ in Denmark had been equally widespread as for COVID-19, the excess COVID-19 mortality gap would likely have been even larger.

Thromboembolic complications in COVID-19 are assumed to be frequent (20). New-onset ischemic stroke was indeed more frequent in COVID-19 than in influenza inpatients. Increased rates of ischemic stroke in COVID-19 compared to influenza were also found in another study based on retrospective medical charts review from 2 academic centers in New York (21). Given that signs and symptoms of stroke - especially minor stroke - may be obscured by systemic illness as well as sedation and ventilation, the true risk may even be higher than the 3- to 7-fold increase reported here and in the cited work (21). We also found that the risk of new-onset diabetes was 3-fold elevated in COVID19-positive individuals compared to negative controls and 2fold elevated compared to influenza-positive patients. These results substantiate concerns of diabetogenic effects of COVID19 (22), including the possibility of ketoacidosis (23). Similarly, nephropathy was frequent in our COVID-19 population, and renal failure may lead to more complications and higher inhospital mortality (24). Ischemic heart disease appeared equally prevalent in inpatients with COVID-19 and those with influenza. Finally, pulmonary embolism occurred more often in our COVID-19 positive population compared to negative controls (albeit not compared to influenza populations).

All these comorbidities, alone or in combination, may put patients with COVID-19 at risk for multiorgan failure. This, together with hypoxemia owing to pulmonary changes, including diffuse alveolar damage with fibrin membranes, thickened alveolar walls, lymphocytic infiltration (25), and pulmonary thrombosis (25), complicated by cardiac arrhythmias, hypotensive shock (26), and possibly brainstem dysfunction (27), is being proposed as the final pathway to death in COVID-19 (28), Many of these mechanisms are unlikely to be specific enough to be reliably captured by diagnostic coding in EHR-based studies such as ours. Large prospective multicenter registries and autopsy studies comparing COVID-19 patients with COVID-19-negative controls and influenza victims are required to dissect the exact contribution of each of these factors.

Concerns for neurological and psychiatric complications in COVID-19 are increasingly being raised (11). Yet, most [albeit not all (10)] reports have revealed a predominance of relatively unspecific symptoms such as altered mental state in highly selected groups without control groups (11, 29, 30), while we report on EHR-registered diagnoses. Our results show decreased or similar frequencies of new-onset neurological and psychiatric diagnoses in COVID-19 individuals within 30 days 


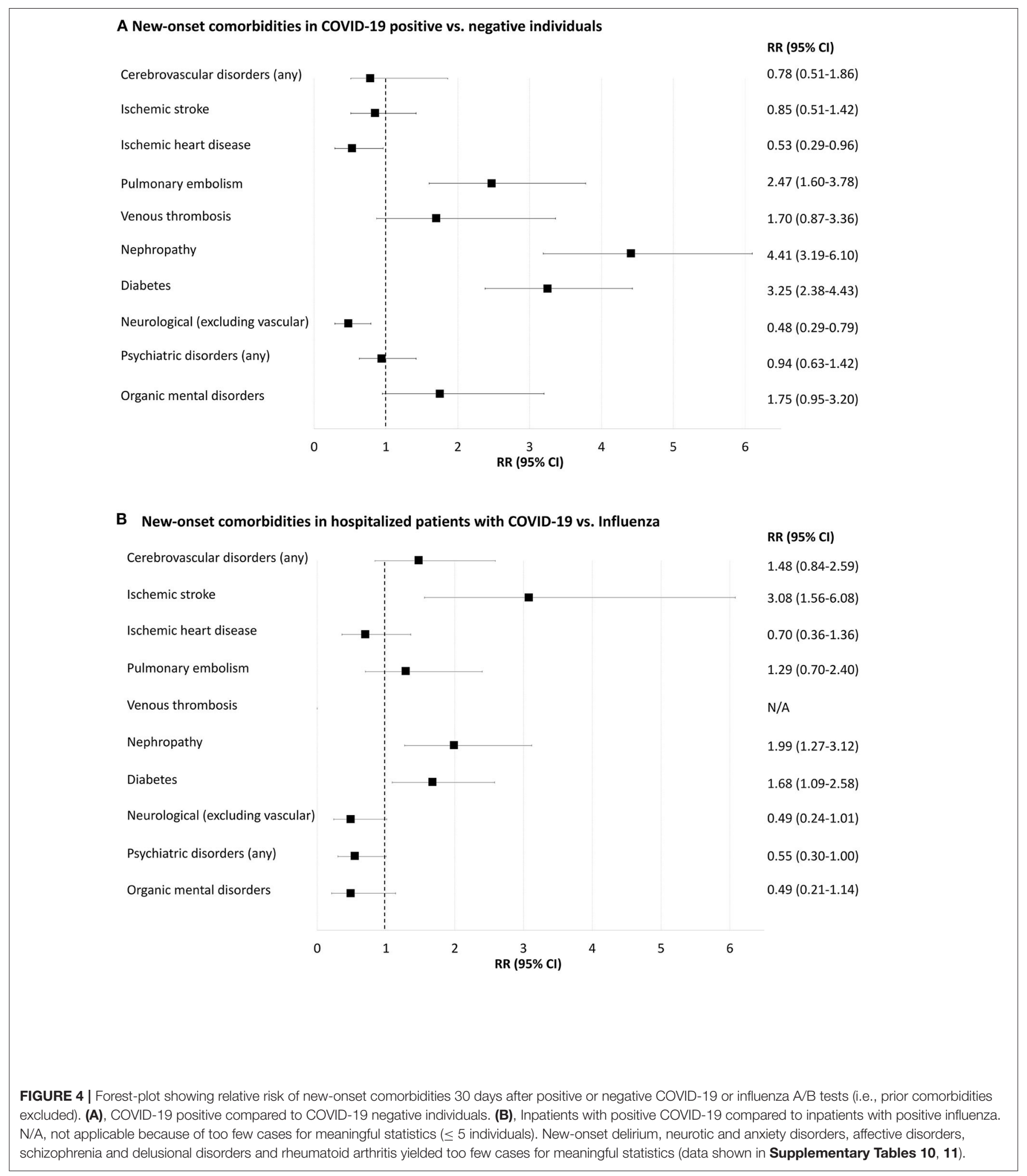

of testing compared to influenza, which suggests either that these complications in COVID-19 are no more frequent than for severe influenza or that the nationwide lockdown in Denmark resulted in fewer contacts to the health care system by people with
COVID-19 but relatively mild comorbid symptoms, including neurological and psychiatric ones. Indeed, observations from California, Italy and Denmark (31-33) indicate a lower incidence of hospitalization of patients with e.g., cardiac disease during 
the COVID-19 lockdown. Further, mild cognitive and emotional symptoms are not likely to be reported within 30 days, and thus the potential long-term consequences of COVID-19 could not be investigated in this study, where we investigated the acute short-term comorbidities.

\section{Strengths and Limitations}

Strengths of our study are related, among others, to the large population numbers and the catchment area-based approach. The extracted general mortality data during years 2018-2020 corresponded well to Danish statistics mortality data (Supplementary Table 2). Numbers of COVID-19 and influenza tests, test results, admissions, and mortality rates in this study were equally consistent with the official Danish numbers (34) Further, test results of SARS-CoV2 and influenza swabs are synchronized with the Danish national microbiology database (13), which is again linked with nationwide mortality data. Therefore, the mortality outcome can be considered virtually complete. We validated our data extraction strategy by ensuring that two individual searches supervised by two independent Epic Slicer-Dicer experts yielded identical results.

As to limitations, we were unable to adjust for confounding factors such as socioeconomics, lifestyle, ethnicity and comorbidities, owing to the use of aggregated EHR data. Instead, we performed stratified analyses according to age- and sexgroups. Of note, people who died of COVID-19 without being tested (i.e., without being recognized as COVID-19 victims) were for obvious reasons not included in our results, which might have led to an underestimation of COVID-19 mortality. Further, we could not adjust for influenza immune prophylaxis given to $10-15 \%$ of the Danish population annually, primarily patients in at risk-groups due to chronic conditions (35). Vaccination reduces influenza rates and increases the chance of a milder course of influenza, leading to a lower probability of new-onset comorbidities and decreased mortality. These effects depend on the effectiveness of the vaccine which varies each year and was particularly low during the 2017/2018 season owing to a mismatch between strains used in the production of the vaccine and those causing the seasonal epidemic (36). In the 2017/2018 peak influenza season, influenza B was identified in $68 \%$ patients tested positive for influenza, while only $17 \%$ were tested positive for influenza A (H3N2) and $14 \%$ for influenza A (H1N1). Vaccine effectiveness was $30-33 \%, 0-13 \%$, and $45-50 \%$ for influenza B, A (H1N1) and A (H3N2), respectively (36). When comparing COVID-19 with influenza $\mathrm{A} / \mathrm{B}$, it must thus be kept in mind that influenza vaccination likely has had a decreasing effect on overall morbidity and mortality in the influenza population. Selection bias might also be considerable because individuals were tested in hospital settings (even as outpatients), and the testing strategy of COVID-19 in Denmark has been much more comprehensive compared to influenza. Furthermore, we only investigated the individuals tested for COVID-19 or influenza, whereas if instead comparing with the entire background population in the capture area, the mortality and morbidity ratios would likely be more increased as the tested population likely have more symptoms and comorbidities than the population not tested.

\section{CONCLUSIONS}

In this first population-based study comparing individuals with COVID-19 positive test results with individuals tested negative for COVID-19 and individuals with influenza, COVID-19 was associated with substantially higher mortality. Due to use of aggregated data with limited ability to adjust for confounders, results must be interpreted with caution, but this mortality is likely even higher than the stated 3.0 to 5.5 -fold increase owing to more extensive testing for COVID-19. In addition, we observed higher rates of new-onset ischemic stroke, diabetes and renal failure. Next, middle- and long-term follow-up data are required to investigate mortality trajectories in COVID-19 vs. influenza populations, and molecular and genetic studies will have to elucidate the specific biological mechanisms behind COVID-19's higher mortality and morbidity compared to influenza.

\section{DATA AVAILABILITY STATEMENT}

The datasets presented in this study can be found in online repositories. The names of the repository/repositories and accession number(s) can be found in the article/Supplementary Material.

\section{AUTHOR CONTRIBUTIONS}

DK and $\mathrm{MB}$ contributed to the conception, design of the study, and contributed equally as senior authors. VN and MA contributed equally as first authors. All authors contributed to the intellectual conception, revision of important intellectual content, and approval of the final version of this manuscript.

\section{FUNDING}

This work received funding from Lundbeckfonden (grant numbers R349-2020-658 and R268-2016-3925), RH Forskningspulje (R143-A6132-B3632), Region Hovedstadens Forskningsfond til Sundhedsforskning 2019 (A6597), Savværksejer Jeppe Juhl og Hustru Ovita Juhls Mindelegat (27062019), and Offerfonden (F-23101-04). The sponsor had no role in the acquisition of the data, interpretation of the results or the decision to publish the findings.

\section{ACKNOWLEDGMENTS}

The authors wish to thank the Slicer Dicer team for their counseling.

\section{SUPPLEMENTARY MATERIAL}

The Supplementary Material for this article can be found online at: https://www.frontiersin.org/articles/10.3389/fmed. 2020.598272/full\#supplementary-material 


\section{REFERENCES}

1. WHO Coronavirus Disease (COVID-19) Dashboard. Available online at: https://covid19.who.int/ (accessed October 06, 2020).

2. COVID-19 Map - Johns Hopkins Coronavirus Resource Center. Available online at: https://coronavirus.jhu.edu/map.html (accessed October 06, 2020).

3. Boretti, A. After less than 2 months, the simulations that drove the world to strict lockdown appear to be wrong, the same of the policies they generated. Heal Serv Res Manag Epidemiol. (2020) 7:233339282093232. doi: 10.1177/2333392820932324

4. WHO | Burden of disease. Available online at: https://www.who.int/influenza/ surveillance_monitoring/bod/en/ (accessed July 10, 2020).

5. Iuliano AD, Roguski KM, Chang HH, Muscatello DJ, Palekar R, Tempia S, et al. Estimates of global seasonal influenzaassociated respiratory mortality: a modelling study. Lancet. (2018) 391:1285-300. doi: 10.1016/S0140-6736(17)33293-2

6. Albini A, Di Guardo G, Noonan DMC, Lombardo M. The SARSCoV-2 receptor, ACE-2, is expressed on many different cell types: implications for ACE-inhibitor- and angiotensin II receptor blocker-based cardiovascular therapies. Intern Emerg Med. (2020) 15:759-66. doi: 10.1007/s11739-020-02364-6

7. Xiong M, Liang X, Wei YD. Changes in blood coagulation in patients with severe coronavirus disease 2019 (COVID-19): a meta-analysis. Br J Haematol. (2020) 189:1050. doi: 10.1111/bjh.16725

8. Mehta P, McAuley DF, Brown M, Sanchez E, Tattersall RS, Manson JJ. COVID19: consider cytokine storm syndromes and immunosuppression. Lancet. (2020) 395:1033-4. doi: 10.1016/S0140-6736(20)30628-0

9. Mallapaty S. Mounting clues suggest the coronavirus might trigger diabetes. Nature. (2020) 583:16-7. doi: 10.1038/d41586-020-01891-8

10. Paterson RW, Brown RL, Benjamin L, Nortley R, Wiethoff S, Bharucha T, et al. The emerging spectrum of COVID-19 neurology: clinical, radiological and laboratory findings. Brain. (2020) 143:3104-20. doi: 10.1093/brain/awaa240

11. Varatharaj A, Thomas N, Ellul MA, Davies NWS, Pollak TA, Tenorio EL, et al. Neurological and neuropsychiatric complications of COVID-19 in 153 patients: a UK-wide surveillance study. Lancet Psychiatr. (2020) 2:18. doi: 10.1016/S2215-0366(20)30287-X

12. ICD-10 Version:2019. Available online at: https://icd.who.int/browse10/2019/ en (accessed July 10, 2020)

13. Voldstedlund M, Haarh M, Mølbak K. The danish microbiology database (MIBA) 2010 to 2013. Eurosurveillance. (2014) 19:20667. doi: 10.2807/1560-7917.es2014.19.1.20667

14. Guan W, Ni Z, Hu Y, Liang W, Ou C, He J, et al. Clinical characteristics of coronavirus disease 2019 in China. N Engl J Med. (2020) 382:170820. doi: 10.1056/NEJMoa2002032

15. Onder G, Rezza G, Brusaferro S. Case-fatality rate and characteristics of patients dying in relation to COVID-19 in Italy. JAMA. (2020) 323:17756. doi: 10.1001/jama.2020.4683

16. Reilev M, Kristensen KB, Pottegård A, Lund LC, Hallas J, Ernst MT, et al. Characteristics and predictors of hospitalization and death in the first 11122 cases with a positive RT-PCR test for SARS-CoV-2 in Denmark: a nationwide cohort. Int J Epidemiol. (2020). doi: 10.1093/ije/ dyaa 140

17. Zhou F, Yu T, Du R, Fan G, Liu Y, Liu Z, et al. Clinical course and risk factors for mortality of adult inpatients with COVID-19 in Wuhan, China: a retrospective cohort study. Lancet. (2020) 395:105462. doi: 10.1016/S0140-6736(20)30566-3

18. Xie J, Covassin N, Fan Z, Singh P, Gao W, Li G, et al. Association between hypoxemia and mortality in patients with COVID-19. Mayo Clin Proc. (2020) 95:1138-47. doi: 10.1016/j.mayocp.2020.04.006

19. Faust JS, del Rio C. Assessment of deaths from COVID19 and from seasonal influenza. JAMA Intern Med. (2020) 180:1045-6. doi: 10.1001/jamainternmed.2020.2306

20. Helms J, Tacquard C, Severac F, Leonard-Lorant I, Ohana M, Delabranche X, et al. High risk of thrombosis in patients with severe SARS-CoV-2 infection: a multicenter prospective cohort study. Intensive Care Med. (2020) 46:108998. doi: 10.1007/s00134-020-06062-X

21. Merkler AE, Parikh NS, Mir S, Gupta A, Kamel H, Lin E, et al. Risk of ischemic stroke in patients with coronavirus disease 2019
(COVID-19) vs. patients with influenza. JAMA Neurol. (2020) Vol 77:136672. doi: 10.1001/jamaneurol.2020.2730

22. Rubino F, Amiel SA, Zimmet P, Alberti G, Bornstein S, Eckel RH, et al. New-onset diabetes in Covid-19. N Engl J Med. (2020) 383:78990. doi: 10.1056/NEJMc2018688

23. Li J, Wang X, Chen J, Zuo X, Zhang H, Deng A. COVID-19 infection may cause ketosis and ketoacidosis. Diabetes Obes Metab. (2020) 22:193541. doi: 10.1111/dom. 14057

24. Uribarri A, Núñez-Gil IJ, Aparisi A, Becerra-Muñoz VM, Feltes G Trabattoni D, et al. Impact of renal function on admission in COVID-19 patients: an analysis of the international HOPE COVID-19 (health outcome predictive evaluation for COVID 19) registry. J Nephrol. (2020) 33:73745. doi: 10.1007/s40620-020-00790-5

25. Edler C, Schröder AS, Aepfelbacher M, Fitzek A, Heinemann A, Heinrich F, et al. Dying with SARS-CoV-2 infection-an autopsy study of the first consecutive 80 cases in Hamburg, Germany. Int J Legal Med. (2020) 134:127584. doi: 10.1007/s00414-020-02317-w

26. Chen Q, Xu L, Dai Y, Ling Y, Mao J, Qian J, et al. Cardiovascular manifestations in severe and critical patients with COVID-19. Clin Cardiol. (2020) 43:796802. doi: $10.1002 /$ clc. 23384

27. Khoo A, McLoughlin B, Cheema S, Weil RS, Lambert C, Manji H, et al. Postinfectious brainstem encephalitis associated with SARS-CoV-2. J Neurol Neurosurg Psychiatr. (2020) 91:1013-4. doi: 10.1136/jnnp-2020323816

28. Fisher HK. Hypoxemia in COVID-19 patients: an hypothesis. Med Hypotheses. (2020) 143:110022. doi: 10.1016/j.mehy.2020.110022

29. Rogers JP, Chesney E, Oliver D, Pollak TA, McGuire P, Fusar-Poli $\mathrm{P}$, et al. Psychiatric and neuropsychiatric presentations associated with severe coronavirus infections: a systematic review and meta-analysis with comparison to the COVID-19 pandemic. Lancet Psychiatr. (2020) 7:61127. doi: 10.1016/S2215-0366(20)30203-0

30. Vindegaard N, Benros ME. COVID-19 pandemic and mental health consequences: systematic review of the current evidence. Brain Behav Immun. (2020) 89:531-42. doi: 10.1016/j.bbi.2020.05.048

31. Solomon MD, McNulty EJ, Rana JS, Leong TK, Lee C, Sung SH, et al. The Covid-19 pandemic and the incidence of acute myocardial infarction. $N$ Engl J Med. (2020) 383:691-3. doi: 10.1056/NEJMc2015630

32. De Filippo O, D'Ascenzo F, Angelini F, Bocchino PP, Conrotto F, et al. Reduced rate of hospital admissions for ACS during Covid-19 outbreak in Northern Italy. N Engl J Med. (2020) 383:88-9. doi: 10.1056/NEJMc2009166

33. Andersson C, Gerds T, Fosbøl E, Phelps M, Andersen J, Lamberts $\mathrm{M}$, et al. Incidence of new-onset and worsening heart failure before and after the COVID-19 epidemic lockdown in Denmark: a nationwide cohort study. Circ Heart Fail. (2020) 13:e007274. doi: 10.1161/CIRCHEARTFAILURE.120.007274

34. Numbers and surveillance of coronavirus/COVID-19 - Danish Health Ministry. Available online at: https://www.sst.dk/da/corona/tal-ogovervaagning\#3 (accessed July 10, 2020).

35. Influenza (age group), Vaccination - Available online at: https://statistik.ssi. $\mathrm{dk} /$ sygdomsdata\#!/?vaccination $=14 \&$ sex $=3$ \&agegroup $=10 \&$ landsdel $=100 \&$ xaxis $=$ Season $\&$ show $=$ Graph \&datatype $=$ Vaccination (accessed August 23, 2020).

36. Influenzasæsonen - opgørelse over sygdomsforekomst 2017/18. Available online at: https://www.ssi.dk/sygdomme-beredskab-ogforskning/sygdomsovervaagning/i/influenzasaesonen--- opgoerelse-oversygdomsforekomst-2017-18 (accessed July 10, 2020).

Conflict of Interest: The authors declare that the research was conducted in the absence of any commercial or financial relationships that could be construed as a potential conflict of interest.

Copyright (c) 2020 Nersesjan, Amiri, Christensen, Benros and Kondziella. This is an open-access article distributed under the terms of the Creative Commons Attribution License (CC BY). The use, distribution or reproduction in other forums is permitted, provided the original author(s) and the copyright owner(s) are credited and that the original publication in this journal is cited, in accordance with accepted academic practice. No use, distribution or reproduction is permitted which does not comply with these terms. 